\title{
Extracellular Matrix-receptor Interaction Signaling Genes Associated with Inferior Breast Cancer Survival
}

\author{
MING-HSIN YEH ${ }^{1,2,3}$, YAU-JIN TZENG ${ }^{4,5}$, TING-YING FU 6 , JUN-JIE YOU ${ }^{4}$, \\ HONG-TAI CHANG ${ }^{1}$, LUO-PING GER ${ }^{4}$ and KUO-WANG TSAI ${ }^{4,5,7}$ \\ ${ }^{1}$ Department of Surgery, Kaohsiung Veterans General Hospital, Kaohsiung, Taiwan, R.O.C.; \\ ${ }^{2}$ Department of Surgery, Chung Shan Medical University Hospital, Taichung, Taiwan, R.O.C.; \\ ${ }^{3}$ School of Medicine, Chung Shan Medical University, Taichung, Taiwan, R.O.C.; \\ ${ }^{4}$ Department of Medical Education and Research, Kaohsiung Veterans General Hospital, Kaohsiung, Taiwan, R.O.C.; \\ ${ }^{5}$ Institute of Biomedical Sciences, National Sun Yat-Sen University, Kaohsiung, Taiwan, R.O.C.; \\ ${ }^{6}$ Department of Pathology and Laboratory Medicine, Kaohsiung Veterans General Hospital, Kaohsiung, Taiwan, R.O.C.; \\ ${ }^{7}$ Department of Chemical Biology, National Pingtung University of Education, Pingtung, Taiwan, R.O.C.
}

\begin{abstract}
Background/Aim: Breast cancer is a common type of cancer in women, and metastasis frequently leads to therapy failure. Using next-generation sequencing (NGS), we aspired to identify the optimal differentially expressed genes (DEGs) for use as prognostic biomarkers for breast cancer. Materials and Methods: NGS was used to determine transcriptome profiles in breast cancer tissues and their corresponding adjacent normal tissues from three patients with breast cancer. Results: Herein, 15 DEGs (fold change $>4$ and $<0.25)$ involved in extracellular matrix $(E C M)$-receptor interaction signaling were identified through NGS. Among them, our data indicated that high HMMR expression levels were correlated with a poor pathological stage $(p<0.001)$ and large tumor size $(p<0.001)$, whereas high COL6A6 and Reelin $($ RELN) expression levels were significantly correlated with an early pathological stage (COL6A6: $p=0.003$ and RELN: $p<0.001)$. Multivariate analysis revealed that high HMMR and SDC1 expression levels were significantly correlated with poor overall survival (OS; HMMR: adjusted hazard ratio [aHR] 1.93, 95\% confidence interval $[\mathrm{CI}]=1.10-3.41$, $p=0.023$; SDC1: [aHR] 2.47, 95\% CI=1.28-4.77, $p=0.007$ ) for breast cancer. Combined, the effects of HMMR and SDC1 showed a significant correlation with poor OS for patients with breast cancer (high expression for both HMMR and SDC1: [aHR] 3.29, 95\%CI=1.52-7.12, $p=0.003)$. Conclusion: These findings suggest that HMMR and SDCl involved in the
\end{abstract}

Correspondence to: Kuo-Wang Tsai, Department of Medical Education and Research, Kaohsiung Veterans General Hospital, Kaohsiung 813, Taiwan, R.O.C. Tel: +886 734221211567, Fax: +886 73468056, e-mail: kwtsai6733@gmail.com

Key Words: Breast cancer, biomarkers, extracellular matrix.
ECM-receptor interaction signaling pathway could act as effective independent prognostic biomarkers for breast ductal carcinoma.

Breast cancer is the most common type of cancer in women worldwide. Although the incidence of breast cancer in some Western countries has recently fallen, its incidence and associated mortality rate continue to increase in Asia (1). Breast cancer is a heterogeneous disease with considerable variation in histological and biological features. Depending on the gene expression signature, breast cancers are classified into four subtypes, namely luminal A, luminal B, epidermal growth factor receptor 2 (HER2), and basal like $(2,3)$. In general, luminal A-type tumors have high estrogen receptor (ER) and progesterone receptor (PR) expression levels and no HER2 expression (ER+/PR+/HER2-), whereas luminal-B type tumors have not only ER and PR expression but also HER2 expression (ER+/PR+/HER2+). Moreover, HER2-type tumors exhibit high HER2 expression but no ER or PR expression (ER-/PR-/HER2+). By contrast, basal-like tumors do not have ER, PR, or HER2 expression (ER-/PR-/HER2-); thus, this type of breast cancer is also called triple-negative breast cancer (TNBC). Studies have revealed that patients with basallike cancer have the least favorable prognosis and 5-year survival, whereas luminal A, luminal B, and HER2 have more favorable prognoses and a well-differentiated cellular morphology (3). The unfavorable prognosis of patients with breast cancer is strongly associated with metastatic events (4$6)$. Furthermore, metastasis is the major cause of cancerrelated deaths, which has been a major challenge in treating breast cancer. Therefore, understanding the mechanism of metastasis and identifying favorable diagnostic and prognostic biomarkers would facilitate improving the survival rate of patients with breast cancer. 
Gene expression signatures are commonly used as biomarkers for predicting breast cancer progression and prognosis $(7,8)$. Next-generation sequencing (NGS) is a powerful, high-throughput technology for comprehensively profiling gene expression and enables for screening of aberrant genes and determining gene signatures for breast cancer $(9,10)$. The Cancer Genome Atlas (TCGA) is an online database that collects substantial NGS gene transcriptome data and clinical data related to breast cancer. These clinical data and information were de-identified and released for research use. Zhong et al. identified the six critical gene signatures by analyzing TCGA database. Furthermore, six-gene expression signatures can be used as independent prognostic markers in survival prediction of patients with breast cancer (11). Using TCGA database, Ong et al. successfully identified a powerful biomarker panel for predicting the prognosis progression of patients with head and neck cancer (12). Therefore, by analyzing these big data, researchers can perform prospective clinical trials and evaluate the impact of candidate genes on breast cancer (13).

In this study, the expression profiles of breast cancer tissue and adjacent normal tissue obtained from three patients were determined by using the NGS approach. Through pathway enrichment analysis, several differentially expressed genes (DEGs) involved in the extracellular matrix (ECM)-receptor interaction signaling pathway were identified. By further analyzing TCGA database, it was determined that HMMR and SDC1 deregulation could be an effective independent prognostic biomarker for breast cancer.

\section{Materials and Methods}

Clinical samples. Clinical samples were collected from patients with breast cancer who had signed an informed consent form and agreed to undergo a surgical procedure at Kaohsiung Veterans General Hospital. This study was approved by the Institutional Review Board (IRB) of Kaohsiung Veterans General Hospital, Kaohsiung, Taiwan (IRB No. VGHKS16-CT10-08). In this study, a total of six clinical samples, comprising three invasive ductal carcinoma (IDC) and three corresponding adjacent normal tissue samples, obtained from three patients with breast cancer were subjected to NGS.

Laser capture microdissection. Fresh-frozen breast cancer specimens were embedded in ornithine carbamyl transferase (Thermo Fisher Scientific Inc., Waltham, MA, USA) stored at $-80^{\circ} \mathrm{C}$ and were then sectioned at a thickness of $10 \mu \mathrm{m}$ and mounted onto MMI-Membrane Slides (Leica Microsystems, Wezlar, Germany). The frozen sections were fixed with ethanol for $30 \mathrm{sec}$ and then stained with hematoxylin and eosin staining before dehydration (30 sec each in $95 \%$ and $100 \%$ ethanol). After air drying, the sections were laser microdissected using an MMI Microdissection Instrument (Olympus, Tokyo, Japan). Laser capture microdissection (LCM) caps were used to collect captured cells from the slices until the caps were filled with tumor cells obtained from a single sample from each patient.
Extraction of RNA from LCM-captured cells. The total RNA of LCM-captured clinical samples was extracted using the miRNeasy Micro Kit (Qiagen, Hilden, Germany) as directed in the instruction manual. Briefly, tissue samples were homogenized in $700 \mu \mathrm{l}$ of QIAzoI Lysis Reagent at room temperature $\left(15^{\circ} \mathrm{C}-25^{\circ} \mathrm{C}\right)$ for $5 \mathrm{~min}$ and mixed with $140 \mu \mathrm{l}$ of chloroform to extract the DNA. The upper aqueous phase was transferred to a new collection tube, and $525 \mu \mathrm{l}$ of $100 \%$ ethanol was added and mixed thoroughly through pipetting. In total, $700 \mu \mathrm{l}$ of sample was added into a RNeasy MinElute spin column and then centrifuged at $\geq 8,000 \times g$ for 15 sec. RNA integrity and quantity were evaluated using a Nanodrop 8000 platform (Thermo Fisher Scientific Inc.).

NGS process. Three high-purity N-T paired tissues were individually separated through LCM and were transferred to an RNA extraction kit (Qiagen) for RNA extraction. The library preparation and NGS processes were performed by a biotechnology company (Genomics, Taipei, Taiwan). Briefly, total RNA was treated with DNase I, and mRNA was further isolated using Oligo(dT) primer. Subsequently, mRNA was further fragmented into small templates for cDNA synthesis. A single nucleotide A (adenine) was then added to the terminal end of the fragments and ligated with adapters. Suitable fragments were selected for polymerase chain reaction (PCR) amplification. After the library preparation was completed, the library quality and concentration were examined using an Agilent 2100 Bioanalyzer and ABI StepOnePlus Real-Time PCR System. Subsequently, the library was sequenced using an Illumina HiSeq 4000 system.

Identification of DEGs. After NGS analysis, raw sequences were obtained from Illumina GA Pipeline software CASAVA Version 1.8 and were expected to generate more than 30 million reads per sample. After low-quality data were filtered, qualified reads were analyzed using TopHat/Cufflinks to estimate gene expression, which was calculated in fragments per kilobase of transcript per million mapped reads (FPKM). For differential expression analyses, CummeRbund was used to statistically analyze the gene expression profiles. The reference genome and gene annotations were retrieved from the Ensembl database. DEGs were screened with the following thresholds: false discovery rate $($ FDR $)<0.001$ and $\log 2$ (fold-change) $>2$ or $<-2$.

Functional annotation of DEGs. DEGs (tumor versus normal $>4$ and $<0.25$ ) were selected from NGS data and then mapped onto the Kyoto Encyclopedia of Genes and Genomes pathways using the Database for Annotation, Visualization, and Integrated Discovery (DAVID). Subsequently, the hypergeometric test was performed to identify significantly enriched pathways.

Expression data from TCGA. From TCGA (https:// cancergenome.nih.gov) data set, 1215 pieces of transcriptome data were downloaded and the corresponding clinical data, including those related to 113 normal cases and 1,102 breast cancer cases. Among these transcriptome profiles, those of 753 cases were included in the clinical pathological features and survival analysis in this study (the expression data of patients were excluded if the patient was male or the tissue type was ductal carcinoma in situ [DCIS]).

Statistical analysis. The paired $t$-test, repeated-measures analysis of variance (ANOVA), and Wilcoxon matched-pairs signed-ranks test were used to compare transcriptome expression levels between the IDC tissues and the adjacent normal tissues. The Mann-Whitney $U$ - 
Table I. Fifteen differentially expressed genes involved in extracellular matrix-receptor interaction are shown.

\begin{tabular}{lcc}
\hline & Up-regulated genes & Down-regulated genes \\
\hline ECM-receptor interaction & COL5A1, COL5A2, COL11A1, COMP, & CD36, ITGA7, RELN, TNXB, \\
& FN1, HMMR, SDC1, SPP1 & ITGA10, LAMC3, COL6A6 \\
\hline
\end{tabular}

test and Kruskal-Wallis one-way ANOVA were used for comparing transcriptome expression levels between different clinicopathologic groups in the tumor tissues. In addition, the log-rank test and Cox proportional hazards model were used to evaluate the effects of different transcriptome expression levels (high $v s$. low) on breast cancer patients' overall survival (OS), disease-free survival, and development of multiple primaries. The difference was considered significant at $p<0.05$.

\section{Results}

Transcriptome profiles of breast cancer. The transcriptome profiles of three patients with breast cancer were obtained through NGS (Figure 1A). Briefly, the subtype of the three cases of breast cancer was determined to be triple-negative type, and the cell morphology was IDC. Three IDC tissues and their corresponding adjacent normal tissues were precisely collected from the three patients through LCM. After RNA extraction and library preparation, the transcriptome profiles of the six samples were determined using the Illumina HiSeq 4000 platform. More than 30 million clean reads were obtained in each library. After mapping the clean reads to the genome, the gene expression abundances were quantified and presented in FPKM. Total of 19,170 genes were identified on average from the sample $([\mathrm{FPKM}]>1)$. Most of these genes were consistently expressed between the adjacent normal group and breast cancer group.

Identification of DEGs. After obtaining the transcriptome profiles, DEGs were identified with the following thresholds: FDR $<0.001$ and fold change (in FPKM values between tumor tissues and the corresponding adjacent normal tissues) $>4$ or $<0.25$. As shown in Figure $1 \mathrm{~A}$, our data revealed that 3219 genes were dysregulated (fold change $>4$ or $<0.25$ ) in tumor tissues compared with normal tissues obtained from patient \#1; among these, 1,914 and 1,305 genes were upregulated and down-regulated, respectively. Moreover, 5158 genes were dysregulated (fold change $>4$ or $<0.25$ ) in tumor tissues compared with normal tissues obtained from patient \#2; among these, 3,256 and 1,902 genes were up-regulated and down-regulated, respectively (Figure 1A). Additionally, 3,824 genes were dysregulated (fold change $>4$ or $<0.25$ ) in tumor tissues compared with normal tissues obtained from patient \#3; among these, 1,392 and 2,432 genes were upregulated and down-regulated, respectively (Figure 1A).
Combining these results revealed that 330 genes were synchronously up-regulated (fold change $>4$ or $<0.25$ and FDR $<0.001$ ) and that 401 genes were down-regulated (fold change $>4$ or $<0.25$ ) in breast tissues compared with the corresponding adjacent normal tissues (Figure 1B).

Analysis of putative biological function of DEGs through pathway enrichment analysis. The aforementioned data revealed that 330 and 401 genes were synchronously upregulated and down-regulated in the three breast cancers, respectively. Subsequently, these DEGs (up-regulation: 330; down-regulation: 401) were subjected to pathway enrichment analysis using the DAVID database. As presented in Figure $1 \mathrm{C}$, these DEGs were significantly enriched in 19 signaling pathways: the ECM-receptor interaction signaling pathway $(p<0.001)$, malaria $(p=0.013)$, staphylococcus aureus infection $(p=0.002)$, lysosome $(p=0.003), \mathrm{ABC}$ transporters $(p=0.003)$, focal adhesion $(p=0.005)$, the PPAR signaling pathway $(p=0.01)$, axon guidance $(p=0.011)$, regulation of lipolysis in adipocytes $(p=0.012)$, protein digestion and absorption $(p=0.016)$, phagosome $(p=0.019)$, hematopoietic cell lineage $(p=0.036)$, biosynthesis of amino acids $(p=0.047)$, the PI3KAkt signaling pathway $(p=0.058)$, proteoglycans in cancer $(p=0.064)$, the Rap1 signaling pathway $(p=0.088)$, the adipocytokine signaling pathway $(p=0.092)$, biosynthesis of antibiotics $(p=0.093)$, and the AMPK signaling pathway $(p=0.095)$. Among them, the ECM-receptor interaction signaling pathway plays a crucial role in modulating breast cancer metastasis (14-16). Therefore, DEGs in the ECMreceptor interaction signaling pathway were prioritized for further examination in this study. As shown in Table I, eight genes (COL5A1, COL5A2, COL11A1, COMP, FN1, HMMR, $S D C 1$, and $S P P 1)$ were significantly up-regulated in breast cancer and enriched in ECM-receptor interaction, whereas seven genes (CD36, COL6A6, ITGA7, ITGA10, LAMC3, $R E L N$, and $T N X B$ ) were significantly down-regulated.

The expression levels of 15 candidate genes were examined in an independent cohort by analyzing TCGA database. A total of 1,215 transcriptome expression profiles and pieces of clinical pathological data were downloaded from TCGA database, comprising 113 adjacent normal tissues and 1,102 breast cancer tumors. As presented in Figure 2 and Table II, the expression levels of the eight genes, namely COL5A1, COL5A2, COL11A1, COMP, FN1, 
A

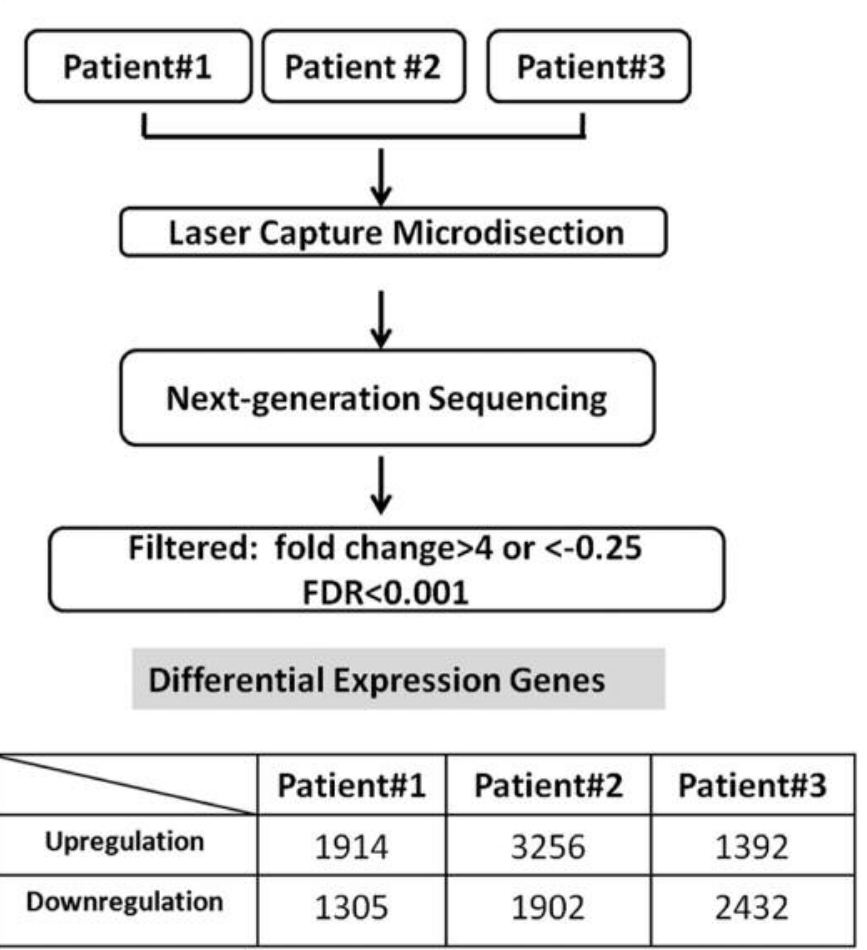

B
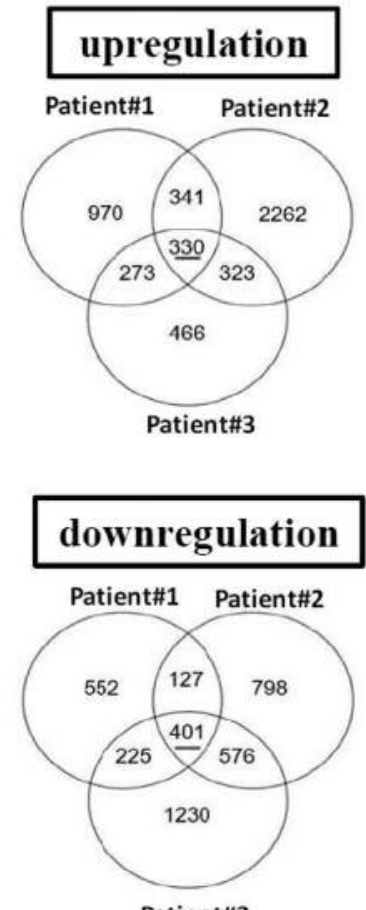

Patient\#3
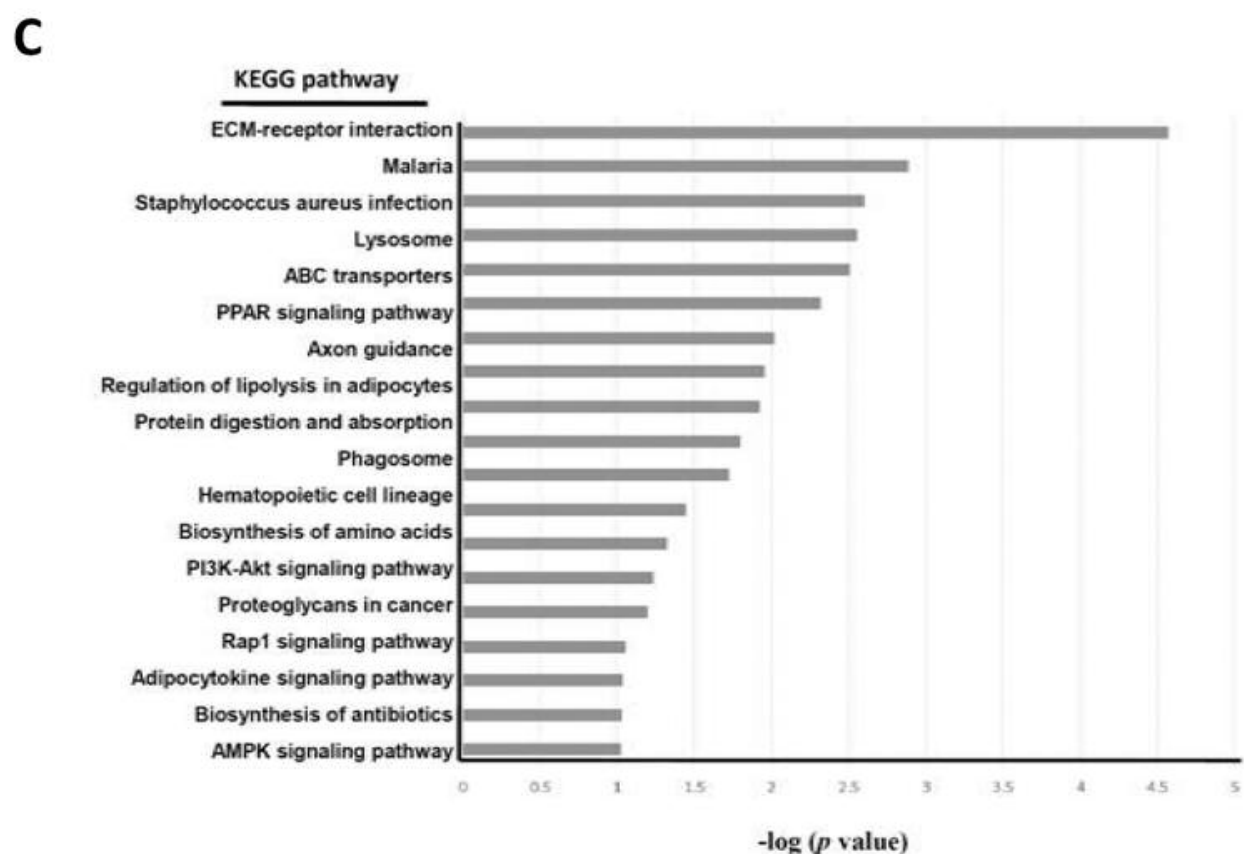

Figure 1. Next-generation sequencing profiles of three N-T-paired breast cancers. (A) Flowchart of identification of differentially expressed genes through next-generation sequencing. Genes with differential expression in breast cancer compared to the adjacent normal samples were filtered with the following thresholds: fold change $\geq 4$ or fold change $<0.25$ and FDR $<0.001$. The numbers of differentially expressed genes in the three patients with breast cancers are respectively showed in the figure panels. (B) Venn diagrams of the number of up-regulated and down-regulated gene candidates in the three N-T-paired breast cancers. (C) Protein coding genes with differential expression were subjected to pathway enrichment analysis (bottom panels). 
A
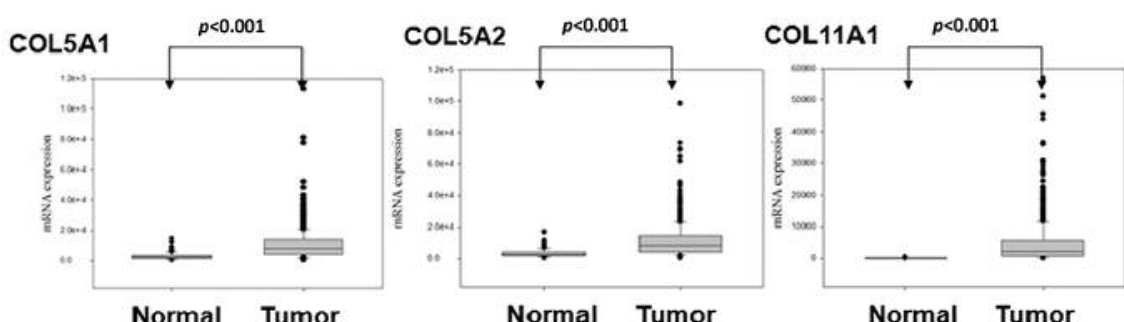

Normal Tumor

Normal Tumor

Normal Tumor
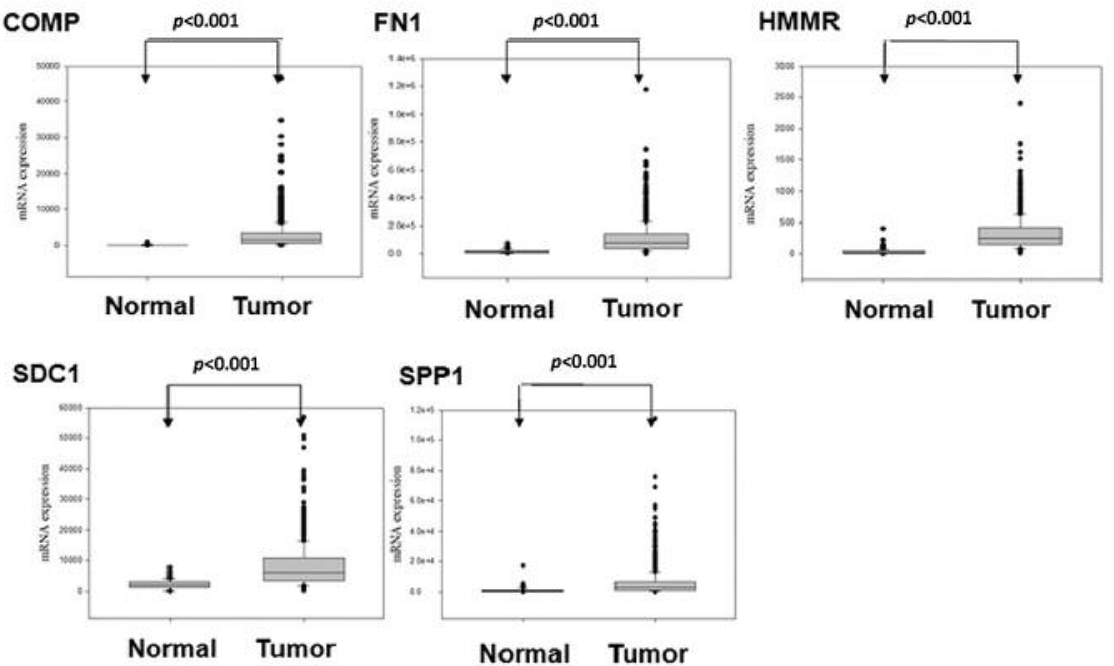

B
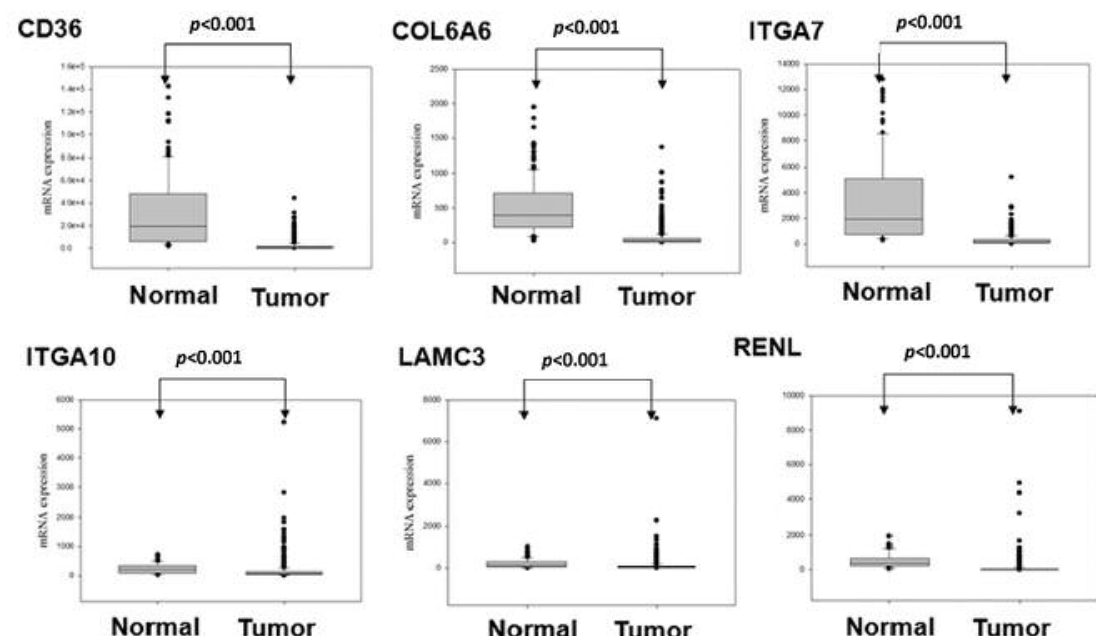

RENL
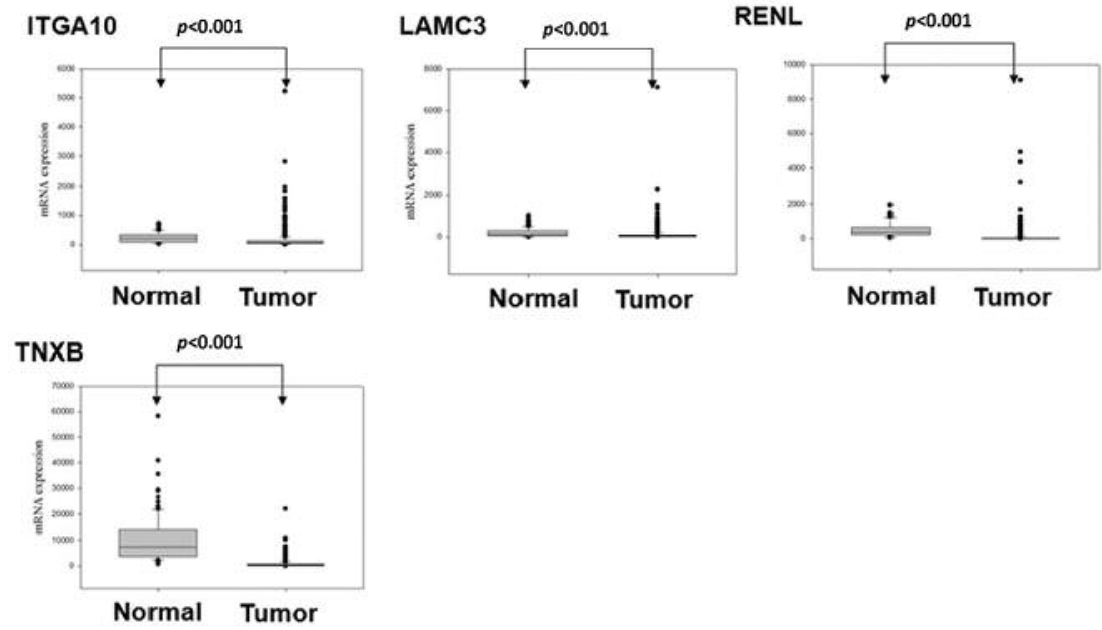

Figure 2. Expression levels of differentially expressed genes involved in the extracellular matrix-receptor interaction signaling pathway were examined by analyzing The Cancer Genome Atlas. (A) and (B) Expression data of eight up-regulated (COL5A1, COL5A2, COL11A1, COMP, FN1, HMMR, SDC1, SPP1) and seven down-regulated genes (CD36, COL6A6, ITGA7, ITGA10, LAMC3, RELN, TNXB) in 1,102 breast cancers compared to 113 corresponding adjacent normal tissues were obtained from The Cancer Genome Atlas. Boxplots of gene expression values (RPKM) for the up-regulated genes in breast cancer are shown. 
Table II. Expression levels of differentially expressed genes analyzed in 1102 breast cancer cases (113 normal vs. 1102 tumor) using The Cancer Genome Atlas.

\begin{tabular}{|c|c|c|c|c|}
\hline \multirow[b]{2}{*}{ Up-regulation } & \multicolumn{2}{|c|}{ TCGA } & \multicolumn{2}{|c|}{ NGS } \\
\hline & Fold change & $p$-Value & Fold change & $p$-Value \\
\hline COL5A1 & 3.8 & $<0.001$ & 14.1 & $<0.001$ \\
\hline COL5A2 & 3.4 & $<0.001$ & 14.0 & $<0.001$ \\
\hline COL11A1 & 93.2 & $<0.001$ & 20.3 & $<0.001$ \\
\hline COMP & 24.1 & $<0.001$ & 14.2 & $<0.001$ \\
\hline FN1 & 7.2 & $<0.001$ & 6.1 & $<0.001$ \\
\hline HMMR & 9.0 & $<0.001$ & 18.6 & $<0.001$ \\
\hline SDC1 & 3.6 & $<0.001$ & 8.0 & $<0.001$ \\
\hline SPP1 & 4.8 & $<0.001$ & 11.2 & $<0.001$ \\
\hline Down-regulation & Fold change & $p$-Value & Fold change & $p$-Value \\
\hline CD36 & 0.1 & $<0.001$ & 0.01 & $<0.001$ \\
\hline ITGA7 & 0.1 & $<0.001$ & 0.03 & $<0.001$ \\
\hline RELN & 0.1 & $<0.001$ & 0.2 & $<0.001$ \\
\hline TNXB & 0.1 & $<0.001$ & 0.07 & $<0.001$ \\
\hline ITGA10 & 0.5 & $<0.001$ & 0.23 & $<0.001$ \\
\hline LAMC3 & 0.4 & $<0.001$ & 0.03 & $<0.001$ \\
\hline COL6A6 & 0.1 & $<0.001$ & 0.02 & $<0.001$ \\
\hline
\end{tabular}

$H M M R, S D C 1$, and $S P P 1$, were significantly increased (fold change $\geq 3$ and $p<0.001$ ) in breast cancer tissues compared with the adjacent normal tissues. Furthermore, the expression levels of seven genes, CD36, COL6A6, ITGA7, ITGA10, $L A M C 3, R E L N$, and $T N X B$, were significantly decreased (fold change $<0.5$ and $p<0.001$ ) in breast cancer compared to adjacent normal tissues. In summary, our data revealed that the expression levels of all DEGs (15 out of 15) in breast cancer that were determined through TCGA database analysis were consistent with our NGS data.

Evaluation of association between DEG expression and survival for breast cancer by Kaplan-Meier plotter. Overall, 15 DEGs involved in the ECM-receptor interaction signaling pathway were identified. To further evaluate the clinical impacts of these DEGs, an online survival analysis tool, the Kaplan-Meier plotter, was used to rapidly assess the effect of the 15 DEGs on breast cancer survival. This database collects the correlation between the survival curve and individual gene expression by analyzing the microarray data of 1809 patients (17). As shown in Table III, the expression levels of four genes, HMMR (hazard ratio [HR] 1.69, 95\% confidence interval $[\mathrm{CI}]=1.36-2.1, p<0.001), \mathrm{SDC} 1(\mathrm{HR}=1.06,95 \% \mathrm{CI}=1.05-1.63$, $p<0.001)$, COL6A6 (HR=0.57, 95\% CI $=0.41-0.79, p<0.001)$, and RELN (HR=0.77, 95\% $\mathrm{CI}=0.62-0.95, p=0.016)$, were significantly correlated with the OS curve of breast cancer (logrank test $p<0.05$ ).
Table III. Evaluation of impact of 15 differentially expressed genes on overall survival of breast cancer by using Kaplan-Meier plotter.

\begin{tabular}{lcc}
\hline \multirow{2}{*}{ ECM-receptor interaction signaling } & \multicolumn{2}{c}{ Overall survival } \\
\cline { 2 - 3 } Gene expression & $p$-Value & Hazard ratio (95\%CI) \\
\hline COL5A1 & 0.6 & $1.06(0.85-1.31)$ \\
COL5A2 & 0.56 & $0.94(0.76-1.16)$ \\
COL11A1 & 0.32 & $1.17(0.86-1.61)$ \\
COMP & 0.8 & $1.03(0.83-1.27)$ \\
FN1 & 0.21 & $1.15(0.93-1.42)$ \\
HMMR & $<0.001$ & $1.69(1.36-2.1)$ \\
SDC1 & $<0.001$ & $1.06(1.05-1.63)$ \\
SPP1 & 0.14 & $0.85(0.69-1.06)$ \\
CD36 & 0.75 & $0.97(0.78-1.2)$ \\
COL6A6 & $<0.001$ & $0.57(0.41-0.79)$ \\
ITGA7 & 0.5 & $0.93(0.75-1.15)$ \\
ITGA10 & 0.29 & $0.89(0.72-1.1)$ \\
LAMC3 & 0.35 & $0.90(0.73-1.12)$ \\
RELN & 0.016 & $0.77(0.62-0.95)$ \\
TNXB & 0.79 & $1.03(0.83-1.27)$ \\
\hline
\end{tabular}

Evaluation of clinical impact of DEGs through TCGA database analysis. The clinical impacts of HMMR, SDC1, RELN, and COL6A6 were further examined by analyzing TCGA database. A total of 753 cases were used in this study to evaluate the correlation between survival and DEG expression (data on male individuals and DCIS were excluded). Using this independent cohort, we confirmed the clinical effects of these four genes in breast cancer. As presented in Table IV, high HMMR expression levels were significantly correlated with a poor American Joint Committee on Cancer (AJCC) pathological stage $(p<0.001)$ and large tumor size $(p<0.001)$, whereas no correlation was observed between clinical pathological features and SDC1 expression. Low COL6A6 expression levels were significantly associated with a high AJCC pathological stage $(p=0.003)$ and large tumor size $(p<0.001$; Table V). High RELN expression levels were significantly correlated with the age of patients with breast cancer $(<40,40-59, v s . \geq 60$ years; $p<0.001)$. Furthermore, low RELN expression levels were highly associated with a poor pathological stage $(p<0.001$; Table V). Notably, our data indicated that the expression levels of HMMR were significantly higher in IDC with the triple-negative subtype compared with those with the luminal A and luminal B subtypes (Table IV and Figure 3). Conversely, higher SDC1 expression levels were observed in breast cancer with Her+ compared with the luminal A, luminal $\mathrm{B}$, and triple-negative subtypes (Table IV and Figure 3). In addition, the expression levels of COL6A6 and RELN did not correlate with any breast cancer subtypes (Table V and Figure 3). These results imply that the expression levels of HMMR and RELN might facilitate classifying breast cancer molecular subtypes. 
Table IV. Correlation of HMMR and SDC1 expression with clinicopathological characteristics of patients with breast cancer.

\begin{tabular}{|c|c|c|c|c|c|c|c|}
\hline \multirow[t]{2}{*}{ Variables } & \multirow[b]{2}{*}{ No. $(\%)$} & \multicolumn{3}{|c|}{ HMMR (n=743) } & \multicolumn{3}{|c|}{ SDC1 $(n=743)$} \\
\hline & & Mean \pm SD & Median & $p$-Value & Mean \pm SD & Median & $p$-Value \\
\hline \multicolumn{8}{|l|}{ Age (yr) } \\
\hline$<40$ & $66(9.0)$ & $383.91 \pm 270.21$ & 284.00 & \multirow[t]{3}{*}{$0.098^{\mathrm{b}}$} & $8605.53 \pm 8191.96$ & 284.00 & \multirow[t]{3}{*}{$0.445^{\mathrm{b}}$} \\
\hline $40-59$ & $354(47.6)$ & $367.04 \pm 242.11$ & 313.50 & & $367.04 \pm 242.11$ & 313.50 & \\
\hline$\geq 60$ & $323(43.5)$ & $331.23 \pm 248.67$ & 268.00 & & $331.23 \pm 248.67$ & 268.00 & \\
\hline \multicolumn{8}{|c|}{ Pathology stage } \\
\hline I & 139 (18.7) & $272.17 \pm 219.51$ & 224.00 & \multirow[t]{4}{*}{$<0.001^{\mathrm{a}}$} & $8143.72 \pm 6826.39$ & 224.00 & \multirow[t]{4}{*}{$0.639^{\mathrm{b}}$} \\
\hline II & $439(59.1)$ & $377.48 \pm 259.65$ & 299.00 & & $8777.75 \pm 7572.85$ & 299.00 & \\
\hline III & $152(20.4)$ & $360.38 \pm 226.64$ & 333.50 & & $9105.76 \pm 6847.86$ & 333.50 & \\
\hline IV & $13(1.8)$ & $302.69 \pm 201.64$ & 237.00 & & $9961.11 \pm 7066.49$ & 237.00 & \\
\hline \multicolumn{8}{|l|}{ pT stage } \\
\hline $\mathrm{T} 1$ & $213(28.6)$ & $277.71 \pm 204.24$ & 244.00 & \multirow[t]{4}{*}{$<0.001^{\mathrm{a}}$} & $8494.48 \pm 6956.80$ & 244.00 & \multirow[t]{4}{*}{$0.930^{\mathrm{b}}$} \\
\hline $\mathrm{T} 2$ & $447(60.2)$ & $378.55 \pm 253.52$ & 305.00 & & 8807.777376 .89 & 305.00 & \\
\hline $\mathrm{T} 3$ & $58(7.8)$ & $451.88 \pm 308.16$ & 354.50 & & $9088.54 \pm 8215.83$ & 354.50 & \\
\hline $\mathrm{T} 4$ & $25(3.4)$ & $307.36 \pm 157.50$ & 288.00 & & $9017.84 \pm 6180.78$ & 288.00 & \\
\hline \multicolumn{8}{|l|}{ pN stage } \\
\hline No & $354(47.7)$ & $343.20 \pm 250.46$ & 286.00 & \multirow[t]{4}{*}{$0.673^{\mathrm{b}}$} & $8579.03 \pm 7209.96$ & 286.00 & \multirow[t]{4}{*}{$0.378^{\mathrm{b}}$} \\
\hline N1 & $264(35.5)$ & $367.86 \pm 263.06$ & 287.5 & & $8627.66 \pm 7620.11$ & 287.5 & \\
\hline $\mathrm{N} 2$ & $90(12.1)$ & $350.69 \pm 214.32$ & 333.5 & & $8963.24 \pm 6424.18$ & 333.5 & \\
\hline N3 & $35(4.7)$ & $345.26 \pm 180.62$ & 309.00 & & 10788.7397 .38 & 309.00 & \\
\hline \multicolumn{8}{|l|}{ pM stage } \\
\hline M0 & $730(98.3)$ & $2804.85 \pm 2059.81$ & 294.00 & \multirow[t]{2}{*}{$0.461^{\mathrm{c}}$} & $8725.32 \pm 7286.07$ & 294.00 & \multirow[t]{2}{*}{$0.544^{\mathrm{c}}$} \\
\hline M1 & $13(1.7)$ & $2384.84 \pm 2044.35$ & 237.00 & & $9961.11 \pm 7066.49$ & 237.00 & \\
\hline \multicolumn{8}{|l|}{ Subtype } \\
\hline Luminal A & $385(51.8)$ & $295.53 \pm 224.21$ & 243.00 & \multirow[t]{5}{*}{$<0.001^{\mathrm{a}}$} & $7488.42 \pm 6187.47$ & 243.00 & \multirow[t]{5}{*}{$<0.001^{\mathrm{a}}$} \\
\hline Luminal B & $103(13.9)$ & $355.50 \pm 215.79$ & 289.00 & & $9367.14 \pm 6475.14$ & 289.00 & \\
\hline Her $2+$ & $33(4.4)$ & $417.21 \pm 222.78$ & 344.00 & & $14573.89 \pm 10466.20$ & 344.00 & \\
\hline TNBC & $132(17.8)$ & $475.64 \pm 279.95$ & 459.50 & & $7886.68 \pm 5802.08$ & 459.50 & \\
\hline unknown & $90(12.1)$ & $392.30 \pm 263.84$ & 317.50 & & $8746.94 \pm 7279.46$ & 317.50 & \\
\hline
\end{tabular}

${ }^{\mathrm{a}} p$-Values were estimated by the Kruskal-Wallis 1-way ANOVA test. ${ }^{\mathrm{b}} p$-Values were estimated by one-way ANOVA test. ${ }^{\mathrm{c}} p$-Values were estimated by the student's T test.

HMMR and SDC1 expressions as independent survival biomarkers. The association of the expression levels of four genes, namely HMMR, SDC1, COL6A6, and RELN, with survival was also evaluated in patients with breast ductal carcinoma. Kaplan-Meier analysis revealed that high HMMR and SDC1 expression levels were significantly correlated with a poor OS curve (HMMR on OS: $p=0.009$ and SCD1 on OS: $p=0.002$; Figure $4 \mathrm{~A}$ and $\mathrm{B}$ ). A multivariate Cox regression model revealed a significant association between high HMMR and SDC1 expression levels with poor OS (HMMR: adjusted $\mathrm{HR}$ [aHR] 1.93, 95\% $\mathrm{CI}=1.10-3.41, \quad p=0.023 ; \quad \mathrm{SDC} 1$ : $\mathrm{aHR}=2.47,95 \% \mathrm{CI}=1.28-4.77, p=0.007$; Table VI).

In addition, high COL6A6 expression levels exhibited a borderline significant association with a satisfactory OS curve of breast cancer ( $p=0.049$; Figure $4 \mathrm{C}$ ), whereas those of RELN exhibited no significant association $(p=0.219$, Figure 4D). The multivariate Cox regression model revealed no significant association between COL6A6 and RELN expression and the OS of patients with breast cancer
(COL6A6: aHR=0.57, 95\%CI=0.29-1.11, $p=0.097$; RELA: aHR=0.76, 95\%CI=0.42-1.40, $p=0.385$; Table VI). Furthermore, the effects of HMMR and SDC1 expression were combined to assess the correlation with survival in breast cancer. High HMMR and SDC1 expression levels were defined as a risk for increasing breast cancer deaths. As shown in Figure 5, high expression levels of both HMMR and SDC1 were associated with significantly shorter survival for patients with breast cancer (OS: $p<0.001)$, even after adjustment for clinicopathologic factors (OS: $\mathrm{aHR}=3.29$, $95 \% \mathrm{CI}=1.52-7.12, p=0.003$; Table VII).

\section{Discussion}

In the present study, 330 up-regulated and 401 down-regulated genes were identified in three TNBC samples through NGS. These DEGs were enriched in several cancer-related signaling pathways. Finally, we focused on ECM-receptor interaction signaling. The ECM comprises proteins and 

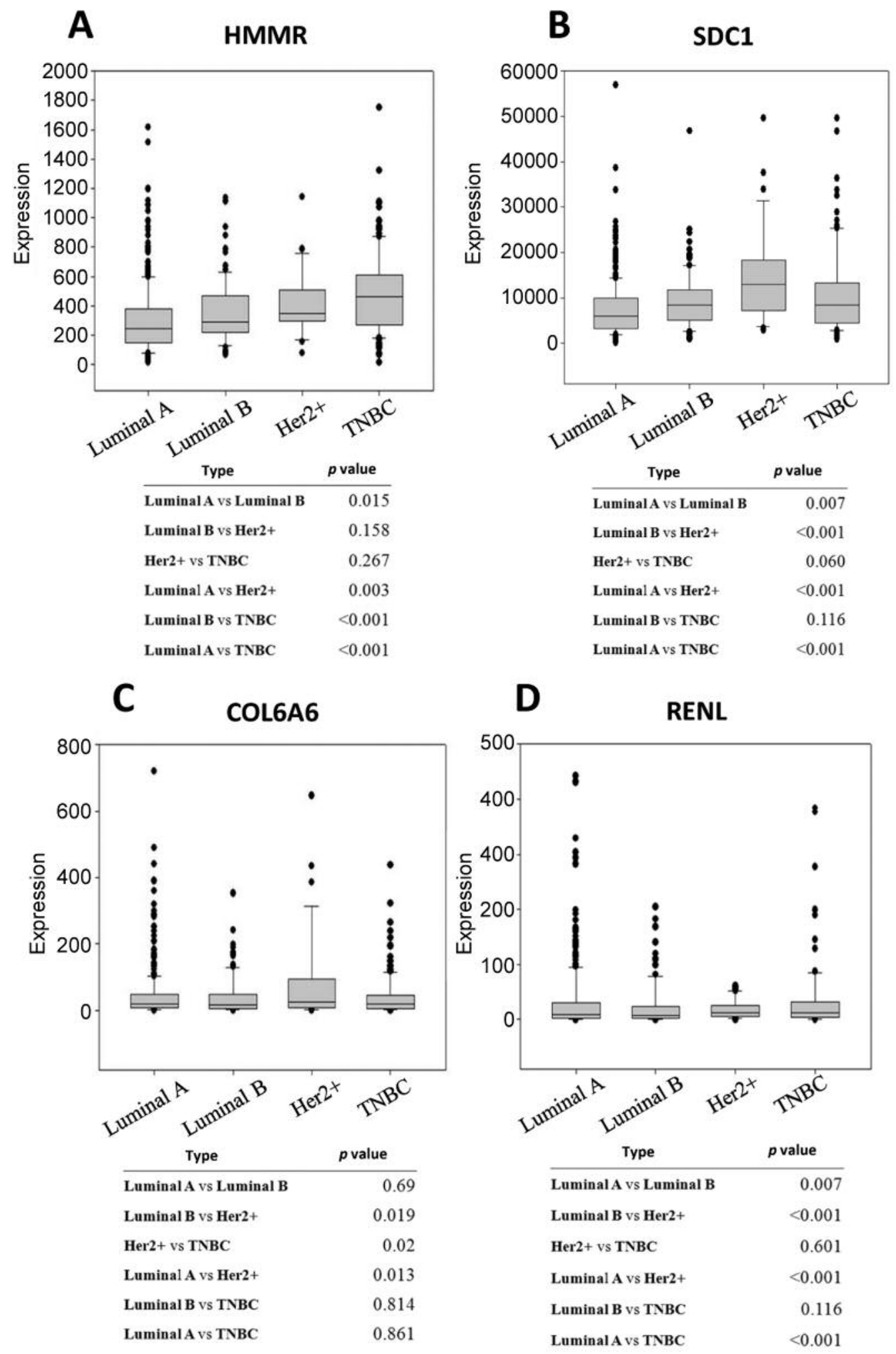

Figure 3. Analysis of the expression levels of HMMR, SDC1, COL6A6, and RELN in specific subtypes of breast cancer. A total of 653 pieces of breast cancer data were downloaded from The Cancer Genome Atlas, comprising of 385 luminal A, 103 luminal B, 33 Her 2 overexpression, and $132 T R B C$, respectively. The expression levels of $(A) H M M R,(B) S D C 1,(C) C O L 6 A 6$, and $(D)$ RELN were evaluated with RPKM by analyzing The Cancer Genome Atlas. 
Table V. Correlation of COL6A6 and RELN expression with clinicopathological characteristics of patients with breast cancer.

\begin{tabular}{|c|c|c|c|c|c|c|c|}
\hline \multirow[t]{2}{*}{ Variables } & \multirow[b]{2}{*}{ No. $(\%)$} & \multicolumn{3}{|c|}{ COL6A6 $(n=743)$} & \multicolumn{3}{|c|}{ RELN (n=743) } \\
\hline & & Mean \pm SD & Median & $p$-Value & Mean \pm SD & Median & $p$-Value \\
\hline \multicolumn{8}{|l|}{ Age (yr) } \\
\hline$<40$ & $66(9.0)$ & $49.55 \pm 64.89$ & 25.49 & \multirow[t]{3}{*}{$0.205^{\mathrm{b}}$} & $53.33 \pm 89.02$ & 25.49 & \multirow[t]{3}{*}{$<0.001^{\mathrm{a}}$} \\
\hline $40-59$ & $354(47.6)$ & $50.16 \pm 88.40$ & 22.10 & & $80.49 \pm 399.83$ & 22.10 & \\
\hline$\geq 60$ & $323(43.5)$ & $39.16 \pm 79.38$ & 15.26 & & $19.146 \pm 38.06$ & 15.26 & \\
\hline \multicolumn{8}{|c|}{ Pathology stage } \\
\hline I & $139(18.7)$ & $65.99 \pm 110.61$ & 30.67 & \multirow[t]{4}{*}{$0.003^{\mathrm{a}}$} & $99.31 \pm 460.96 \mathrm{e}$ & 30.67 & \multirow[t]{4}{*}{$<0.001^{\mathrm{a}}$} \\
\hline II & $439(59.1)$ & $37.42 \pm 60.25$ & 16.69 & & $41.88 \pm 249.07$ & 16.69 & \\
\hline III & $152(20.4)$ & $43.90 \pm 69.45$ & 17.93 & & $33.93 \pm 66.60$ & 17.93 & \\
\hline IV & $13(1.8)$ & $107.93 \pm 279.69$ & 12.48 & & $65.42 \pm 199.74$ & 12.48 & \\
\hline \multicolumn{8}{|l|}{ pT stage } \\
\hline $\mathrm{T} 1$ & $213(28.6)$ & $64.02 \pm 103.45$ & 27.26 & \multirow[t]{4}{*}{$<0.001^{\mathrm{a}}$} & $77.37 \pm 374.80$ & 27.26 & \multirow[t]{4}{*}{$0.427^{\mathrm{b}}$} \\
\hline $\mathrm{T} 2$ & $447(60.2)$ & $38.38 \pm 73.37$ & 16.23 & & $43.05 \pm 249.04$ & 16.23 & \\
\hline $\mathrm{T} 3$ & $58(7.8)$ & $37.22 \pm 65.38$ & 14.27 & & $34.61 \pm 80.98$ & 14.27 & \\
\hline $\mathrm{T} 4$ & $25(3.4)$ & $29.06 \pm 51.52$ & 12.76 & & $18.75 \pm 27.05$ & 12.76 & \\
\hline \multicolumn{8}{|l|}{$\mathrm{pN}$ stage } \\
\hline No & $354(47.7)$ & $46.17 \pm 83.32$ & 17.41 & \multirow[t]{4}{*}{$0.787^{b}$} & $67.64 \pm 396.09$ & 17.41 & \multirow[t]{4}{*}{$0.425^{\mathrm{a}}$} \\
\hline N1 & $264(35.5)$ & $43.75 \pm 86.54$ & 19.51 & & $39.21 \pm 87.29$ & 19.51 & \\
\hline $\mathrm{N} 2$ & $90(12.1)$ & $41.84 \pm 71.74$ & 16.98 & & $32.38 \pm 66.04$ & 16.98 & \\
\hline N3 & $35(4.7)$ & $57.60 \pm 76.32$ & 25.19 & & $28.18 \pm 52.40$ & 25.19 & \\
\hline \multicolumn{8}{|l|}{ pM stage } \\
\hline M0 & $730(98.3)$ & $44.21 \pm 74.93$ & 18.28 & \multirow[t]{2}{*}{$0.420^{\mathrm{d}}$} & $51.16 \pm 281.01$ & 18.28 & \multirow[t]{2}{*}{$0.856^{\mathrm{c}}$} \\
\hline M1 & $13(1.7)$ & $107.93 \pm 279.69$ & 12.48 & & $65.42 \pm 199.74$ & 12.48 & \\
\hline \multicolumn{8}{|l|}{ Subtype } \\
\hline Luminal A & $385(51.8)$ & $44.54 \pm 77.68$ & 18.85 & \multirow[t]{5}{*}{$0.705^{\mathrm{a}}$} & $46.51 \pm 235.60$ & 18.85 & \multirow[t]{5}{*}{$0.507^{\mathrm{a}}$} \\
\hline Luminal B & $103(13.9)$ & $41.25 \pm 60.00$ & 16.48 & & $24.03 \pm 40.42$ & 16.48 & \\
\hline Her $2+$ & $33(4.4)$ & $83.04 \pm 144.01$ & 26.25 & & $18.62 \pm 17.51$ & 26.25 & \\
\hline TNBC & $132(17.8)$ & $43.21 \pm 66.01$ & 19.08 & & $99.16 \pm 515.89$ & 19.08 & \\
\hline Unknown & $90(12.1)$ & $42.64 \pm 111.90$ & 17.23 & & $45.66 \pm 119.39$ & 17.23 & \\
\hline
\end{tabular}

${ }^{\mathrm{a}} p$-values were estimated by Kruskal-Wallis 1 -way ANOVA test. ${ }^{\mathrm{b}} p$-Values were estimated by one-way ANOVA test. ${ }^{\mathrm{c} p}$-Value were estimated by student's T test. ${ }^{\mathrm{d}} p$-Value were estimated by Mann-Whitney U-T test.

proteoglycans/glycosaminoglycans that provide structural support in human cells. Studies have shown that dysregulation of ECM components plays a crucial role in promoting cancer cell invasion and progression (14-16). HMMR are major components of the ECM and are involved in regulated cancer cell growth, migration, and invasion (16). In this study, our data reveal that HMMR overexpression was highly correlated with poor pathological stages and poor survival rates. Previous studies have revealed that HMMR is a multifunctional extracellular and intracellular protein and can regulate cell motility and the cell cycle (18). Stevens et al. reported that HMMR overexpression in primary lung adenocarcinoma was associated with poor prognosis (19). Akent'eva et al. showed that breast cancer cell RHAMM-selective peptides can induce apoptosis and necrosis of breast cancer cells, implying that HMMR-selective peptides have potential as a target therapy for cancer (20). Wang et al. reported that RHAMM overexpression was observed in breast cancer and that high RHAMM expression was significantly associated with poor prognostic value (21).
SDC1 belongs to the syndecan family and comprises cell surface heparan sulfate proteoglycans (HSPGs) (22). SDC1 has been demonstrated to play a critical role in regulating ECM architecture and facilitating breast cancer invasion and progression (23-25). Numerous studies have revealed that SDC1 was frequently down-regulated in most human cancers, including colon cancer, head and neck cancer, and gastric cancer (26-29). However, the expression levels of SDC1 have been reported to increase in breast cancer and pancreatic cancer $(30,31)$. A previous study reported that SDC1overexpression was involved in promoting cell growth and cell invasion ability in breast cancer (32). Furthermore, SDC1 knockdown could interrupt colony and spheroid formation by regulating cancer stem cell-related genes in breast cancer (33). In this study, SDC1 was significantly up-regulated in breast cancer and high SDC1 expression was positively associated with poor survival of patients with breast cancer. Our results revealed that SDC1 might play an oncogenic role in regulating breast cancer progression. Previous studies have 

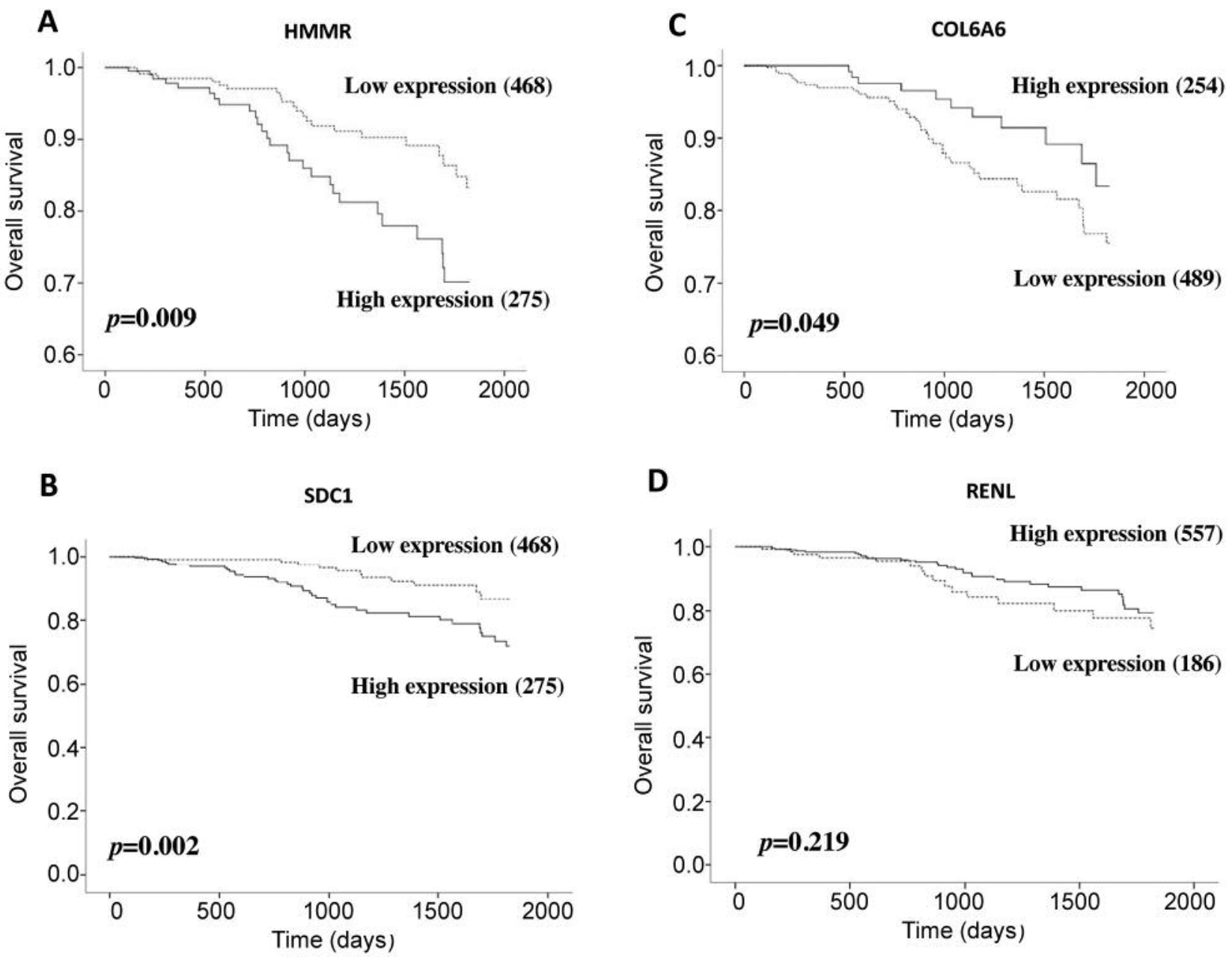

Figure 4. Analysis of the prognostic significance of HMMR, SDC1, COL6A6, and RELN in breast cancer. (A-D) A total of 743 overall survival curves were assessed depending on the HMMR, SDC1, COL6A6, and RELN expression levels in breast cancer tissues.

Table VI. Univariate and multivariate Cox regression analysis of gene expression for overall survival of patients with breast cancer patient.

\begin{tabular}{|c|c|c|c|c|c|}
\hline \multirow[t]{2}{*}{ Characteristic } & \multirow{2}{*}{$\begin{array}{l}\text { No. }(\%) \\
(n=743)\end{array}$} & \multicolumn{4}{|c|}{ OS } \\
\hline & & CHR $(95 \% \mathrm{CI})$ & $p$-Value & AHR $(95 \% \mathrm{CI})$ & $p$-Value \\
\hline \multicolumn{6}{|l|}{ HMMR } \\
\hline Low & $468(63.0)$ & 1.00 & & 1.00 & \\
\hline High & $275(37.0)$ & $2.07(1.19-3.61)$ & 0.010 & $1.93(1.10-3.41)$ & 0.023 \\
\hline \multicolumn{6}{|l|}{ SDC1 } \\
\hline Low & $316(42.5)$ & 1.00 & & 1.00 & \\
\hline High & $427(57.5)$ & $2.66(1.39-5.09)$ & 0.003 & $2.47(1.28-4.77)$ & 0.007 \\
\hline \multicolumn{6}{|l|}{ COL6A6 } \\
\hline Low & $489(65.8)$ & 1.00 & & 1.00 & \\
\hline High & $254(34.2)$ & $0.52(0.26-1.01)$ & 0.053 & $0.57(0.29-1.11)$ & 0.097 \\
\hline \multicolumn{6}{|l|}{ RELN } \\
\hline Low & $186(25.0)$ & 1.00 & & 1.00 & \\
\hline High & $557(75.0)$ & $0.69(0.38-1.25)$ & 0.220 & $0.76(0.42-1.40)$ & 0.385 \\
\hline
\end{tabular}

DSS: Disease-specific survival; DFS: disease-free survival; CHR: crude hazard ratio; AHR: adjusted hazard ratio; OS: overall survival. AHR were adjusted for AJCC pathological stage (II, III and IV vs. I). 


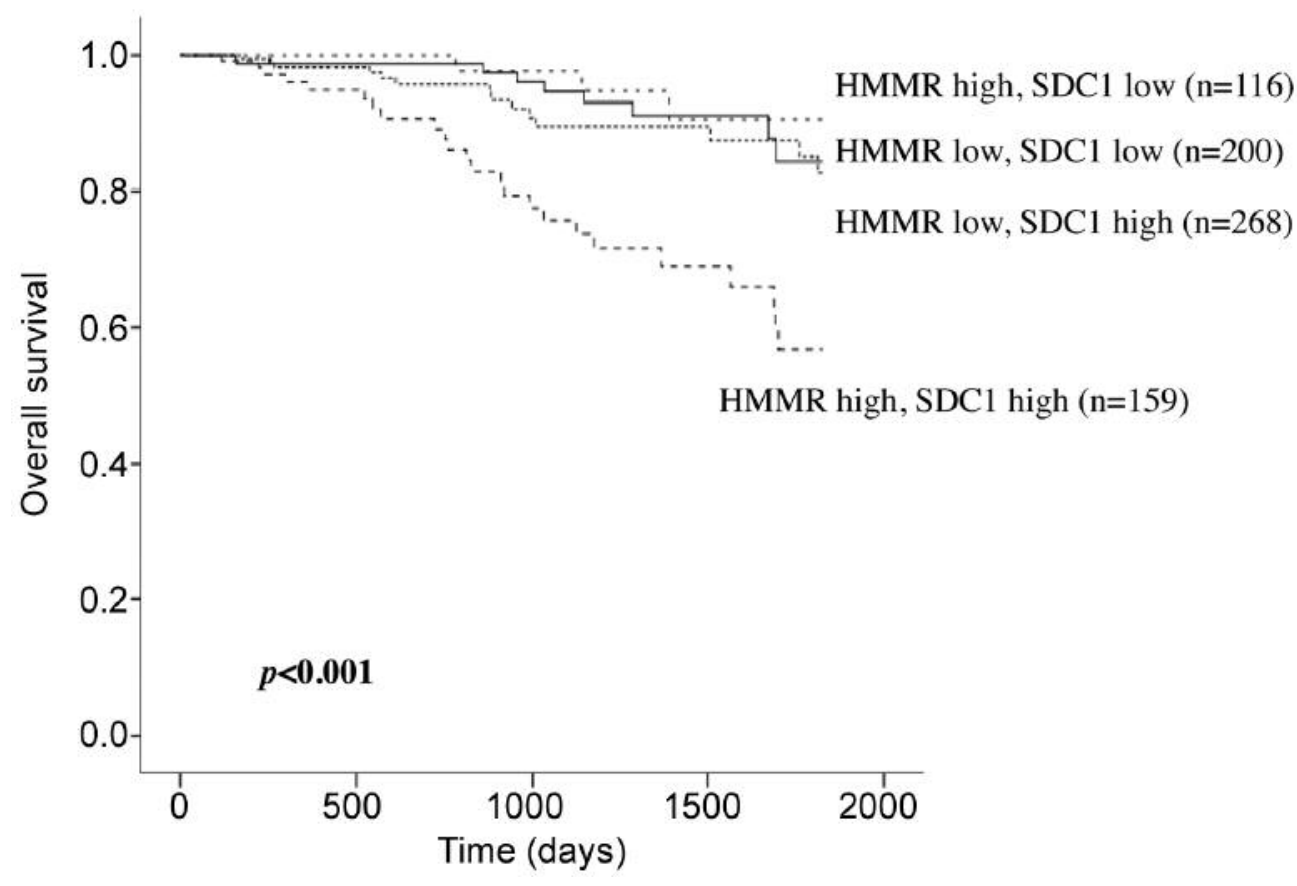

Figure 5. Combination of HMMR and SDC1 expression levels to analyze their prognostic significance in breast cancer. Overall survival was compared according to the combined HMMR and SDC1 expression in breast cancer.

Table VII. Univariate and multivariate Cox's regression analysis of HMMR and SDC1 for overall survival of breast cancer patients.

\begin{tabular}{|c|c|c|c|c|c|}
\hline \multirow[t]{2}{*}{ Risk } & \multirow{2}{*}{$\begin{array}{c}\text { No. }(\%) \\
N=743\end{array}$} & \multicolumn{4}{|c|}{ OS } \\
\hline & & CHR $(95 \% \mathrm{CI})$ & $p$-Value & AHR $(95 \%$ CI $)$ & $p$-Value \\
\hline HMMR low, SDC1 low & $200(26.9)$ & 1.00 & & 1.00 & \\
\hline HMMR high, SDC1 low & $116(15.6)$ & $0.59(0.16-2.19)$ & 0.431 & $0.52(0.14-1.95)$ & 0.335 \\
\hline HMMR low, SDC1 high & $268(36.1)$ & $1.31(0.57-3.03)$ & 0.526 & $1.18(0.50-2.75)$ & 0.710 \\
\hline HMMR high, SDC1 high & $159(21.4)$ & $3.94(1.83-8.47)$ & $<0.001$ & $3.29(1.52-7.12)$ & 0.003 \\
\hline
\end{tabular}

OS: Overall survival; CI: confidence interval; CHR: crude hazard ratio; AHR: adjusted hazard ratio. AHR were adjusted for AJCC pathological stage (II, III and IV vs. I).

reported similar results that SDC1 was significantly upregulated in breast cancer and that high SDC1 expression was correlated with poor prognostic value for breast cancer (31, 34, 35). Nguyen et al. reported that high SDC1 expression was significantly associated with a higher histological grade and had a negative effect on both OS and disease-free survival for patients with breast cancer (35). In addition, soluble SDC1 was reported to be detectable in sera, and SDC1 levels were increased in the sera of patients with breast cancer compared to healthy controls; moreover, high SDC1 levels were highly associated with breast tumor sizes (36). Overall, SDC1 could not only be a diagnostic biomarker, but also a prognostic biomarker for breast cancer.
In conclusion, the present study determined gene expression profiles and identified 15 DEGs involved in the ECM-receptor interaction signaling pathway. Using the TCGA database, further evaluation was executed and it was observed that two genes, $H M M R$ and $S D C 1$, can be used as an effective independent prognostic biomarkers in survival prediction for breast ductal carcinomas.

\section{Acknowledgements}

This work was supported by funding from the Ministry of Science Technology (MOST 106-2320-B-075B-004) and Kaohsiung Veterans General Hospital (VGHKS 105-072 and VGHKS105-135). 


\section{Conflicts of Interest}

The Authors declare that there are no conflicts of interest to disclose.

\section{References}

1 Porter PL: Global trends in breast cancer incidence and mortality. Salud Publica Mex 51: s141-146, 2009.

2 Alco G, Bozdogan A, Selamoglu D, Pilanci KN, Tuzlali S, Ordu C, Igdem S, Okkan S, Dincer M, Demir G and Ozmen V: Clinical and histopathological factors associated with Ki-67 expression in breast cancer patients. Oncol Lett 9: 1046-1054, 2015.

3 Goldhirsch A, Wood WC, Coates AS, Gelber RD, Thurlimann B and Senn HJ: Strategies for subtypes - dealing with the diversity of breast cancer: highlights of the St. Gallen International Expert Consensus on the Primary Therapy of Early Breast Cancer 2011. Ann Oncol 22: 1736-1747, 2011.

4 Mehlen P and Puisieux A: Metastasis: a question of life or death. Nat Rev Cancer 6: 449-458, 2006.

5 Monteiro J and Fodde R: Cancer stemness and metastasis: therapeutic consequences and perspectives. Eur J Cancer 46: 1198-1203, 2010.

6 Nguyen DX and Massague J: Genetic determinants of cancer metastasis. Nat Rev Genet 8: 341-352, 2007.

7 van de Vijver MJ, He YD, van't Veer LJ, Dai H, Hart AA, Voskuil DW, Schreiber GJ, Peterse JL, Roberts C, Marton MJ, Parrish M, Atsma D, Witteveen A, Glas A, Delahaye L, van der Velde T, Bartelink H, Rodenhuis S, Rutgers ET, Friend $\mathrm{SH}$ and Bernards R: A gene-expression signature as a predictor of survival in breast cancer. N Engl J Med 347: 1999-2009, 2002.

8 Paik S, Tang G, Shak S, Kim C, Baker J, Kim W, Cronin M, Baehner FL, Watson D, Bryant J, Costantino JP, Geyer CE, Jr., Wickerham DL and Wolmark N: Gene expression and benefit of chemotherapy in women with node-negative, estrogen receptorpositive breast cancer. J Clin Oncol 24: 3726-3734, 2006.

9 Iyer MK, Chinnaiyan AM and Maher CA: ChimeraScan: a tool for identifying chimeric transcription in sequencing data. Bioinformatics 27: 2903-2904, 2011.

10 Ma L, Nie L, Liu J, Zhang B, Song S, Sun M, Yang J, Yang Y, Fang $\mathrm{X}, \mathrm{Hu} \mathrm{S}$, Zhao Y and Yu J: An RNA-seq-based gene expression profiling of radiation-induced tumorigenic mammary epithelial cells. Genomics Proteomics Bioinformatics 10: 326-335, 2012.

11 Zhong L, Lou G, Zhou X, Qin Y, Liu L and Jiang W: A six-long non-coding RNAs signature as a potential prognostic marker for survival prediction of ER-positive breast cancer patients. Oncotarget 8: 68861-67870, 2017.

12 Ong CJ, Shannon NB, Mueller S, Lek SM, Qiu X, Chong FT, Li K, Koh KKN, Tay GCA, Skanthakumar T, Hwang JSG, Lim TKH, Ang MK, Tan DSW, Tan NC, Tan HK, Soo KC and Iyer NG: A three gene immunohistochemical panel serves as an adjunct to clinical staging of patients with head and neck cancer. Oncotarget 8: 79556-79566, 2017.

13 Kim SY, Kawaguchi T, Yan L, Young J, Qi Q and Takabe K: Clinical relevance of microRNA expressions in breast cancer validated using the Cancer Genome Atlas (TCGA). Ann Surg Oncol 24: 2943-2949, 2017.
14 Huan JL, Gao X, Xing L, Qin XJ, Qian HX, Zhou Q and Zhu L: Screening for key genes associated with invasive ductal carcinoma of the breast via microarray data analysis. Genet Mol Res 13: 7919-7925, 2014.

15 Schwertfeger KL, Cowman MK, Telmer PG, Turley EA and McCarthy JB: Hyaluronan, Inflammation, and Breast Cancer Progression. Front Immunol 6: 236, 2015.

16 Tolg C, McCarthy JB, Yazdani A and Turley EA: Hyaluronan and RHAMM in wound repair and the "cancerization" of stromal tissues. Biomed Res Int 2014: 103923, 2014.

17 Gyorffy B, Lanczky A, Eklund AC, Denkert C, Budczies J, Li $\mathrm{Q}$, and Szallasi Z: An online survival analysis tool to rapidly assess the effect of 22,277 genes on breast cancer prognosis using microarray data of 1,809 patients. Breast Cancer Res Treat 123: 725-731, 2010.

18 Sohr S and Engeland K: RHAMM is differentially expressed in the cell cycle and down-regulated by the tumor suppressor p53. Cell Cycle 7: 3448-3460, 2008.

19 Stevens LE, Cheung WKC, Adua SJ, Arnal-Estape A, Zhao M, Liu Z, Brewer K, Herbst RS, and Nguyen DX: Extracellular matrix receptor expression in subtypes of lung adenocarcinoma potentiates outgrowth of micrometastases. Cancer Res 77: 19051917, 2017.

20 Akent'eva NP, Shushanov SS and Kotel'nikov AI: Effects of RHAMM/HMMR-Selective Peptides on Survival of Breast Cancer Cells. Bull Exp Biol Med 159: 658-661, 2015.

21 Wang C, Thor AD, Moore DH, 2nd, Zhao Y, Kerschmann R, Stern R, Watson PH and Turley EA: The overexpression of RHAMM, a hyaluronan-binding protein that regulates ras signaling, correlates with overexpression of mitogen-activated protein kinase and is a significant parameter in breast cancer progression. Clin Cancer Res 4: 567-576, 1998.

22 Choi Y, Chung H, Jung H, Couchman JR and Oh ES: Syndecans as cell surface receptors: Unique structure equates with functional diversity. Matrix Biol 30: 93-99, 2011.

23 Yang N, Mosher R, Seo S, Beebe D and Friedl A: Syndecan-1 in breast cancer stroma fibroblasts regulates extracellular matrix fiber organization and carcinoma cell motility. Am J Pathol 178: 325-335, 2011.

24 Stanley MJ, Stanley MW, Sanderson RD and Zera R: Syndecan1 expression is induced in the stroma of infiltrating breast carcinoma. Am J Clin Pathol 112: 377-383, 1999.

25 Maeda T, Alexander CM and Friedl A: Induction of syndecan-1 expression in stromal fibroblasts promotes proliferation of human breast cancer cells. Cancer Res 64: 612-621, 2004.

26 Peretti T, Waisberg J, Mader AM, de Matos LL, da Costa RB, Conceicao GM, Lopes AC, Nader HB and Pinhal MA: Heparanase2, syndecan-1, and extracellular matrix remodeling in colorectal carcinoma. Eur J Gastroenterol Hepatol 20: 756-765, 2008.

27 Inki P, Joensuu H, Grenman R, Klemi $\mathrm{P}$ and Jalkanen M: Association between syndecan-1 expression and clinical outcome in squamous cell carcinoma of the head and neck. Br J Cancer 70: 319-323, 1994.

28 Pap Z, Pavai Z, Denes L, Kovalszky I and Jung J: An immunohistochemical study of colon adenomas and carcinomas: Ecadherin, Syndecan-1, Ets-1. Pathol Oncol Res 15: 579-587, 2009.

$29 \mathrm{Hu}$ XF, Yao J, Gao SG, Yang YT, Peng XQ and Feng XS: Midkine and syndecan 1 levels correlate with the progression of malignant gastric cardiac adenocarcinoma. Mol Med Rep 10: 1409-1415, 2014. 
30 Conejo JR, Kleeff J, Koliopanos A, Matsuda K, Zhu ZW, Goecke H, Bicheng N, Zimmermann A, Korc M, Friess $\mathrm{H}$ and Buchler MW: Syndecan-1 expression is up-regulated in pancreatic but not in other gastrointestinal cancers. Int $\mathrm{J}$ Cancer 88: 12-20, 2000.

31 Cordone I, Masi S, Summa V, Carosi M, Vidiri A, Fabi A, Pasquale A, Conti L, Rosito I, Carapella CM, Villani V and Pace A: Overexpression of syndecan-1, MUC-1, and putative stem cell markers in breast cancer leptomeningeal metastasis: a cerebrospinal fluid flow cytometry study. Breast Cancer Res 19: 46, 2017.

32 Ibrahim SA, Yip GW, Stock C, Pan JW, Neubauer C, Poeter M, Pupjalis D, Koo CY, Kelsch R, Schule R, Rescher U, Kiesel L and Gotte M: Targeting of syndecan-1 by microRNA miR-10b promotes breast cancer cell motility and invasiveness via a RhoGTPase- and E-cadherin-dependent mechanism. Int J Cancer 131: E884-896, 2012.

33 Ibrahim SA, Gadalla R, El-Ghonaimy EA, Samir O, Mohamed HT, Hassan H, Greve B, El-Shinawi M, Mohamed MM and Gotte M: Syndecan-1 is a novel molecular marker for triple negative inflammatory breast cancer and modulates the cancer stem cell phenotype via the IL-6/STAT3, Notch and EGFR signaling pathways. Mol Cancer 16: 57, 2017.
34 Lim GH, Tan PH, Jara-Lazaro AR, Thike AA, Sim WC, Yap VB and Yip GW: Syndecan-1 is a potential biomarker for triplepositive breast carcinomas in Asian women with correlation to survival. Singapore Med J 55: 468-472, 2014.

35 Nguyen TL, Grizzle WE, Zhang K, Hameed O, Siegal GP and Wei S: Syndecan-1 overexpression is associated with nonluminal subtypes and poor prognosis in advanced breast cancer. Am J Clin Pathol 140: 468-474, 2013.

36 Malek-Hosseini Z, Jelodar S, Talei A, Ghaderi A and Doroudchi M: Elevated Syndecan-1 levels in the sera of patients with breast cancer correlate with tumor size. Breast Cancer 24: 742-747, 2017.
Received May 7, 2018

Revised June 13, 2018

Accepted June 20, 2018 